\title{
Quality Metrics for Color Images
}

\author{
Gwanggil Jeon \\ Department of Embedded Systems Engineering, Incheon National University \\ 119 Academy-ro, Yeonsu-gu, Incheon 406-772, Korea \\ gjeon@inu.ac.kr
}

\begin{abstract}
In this paper, image quality assessment and comparison are provided. The goal of this study is to assess image without the association of human viewers. The image quality weights the received image distortion compare to the original image. Four metrics are explained and assessed in the experimental results. They are color mean squared error (CMSE), color peak signal to noise ratio (CPSNR), spatial-CIELAB (SCIELAB), and feature SIMilarity (FSIM). Simulation results show restored images obtained by different factor values. The results are shown in several metrics, numerical results in terms of CMSE, CPSNR, S-CIELAB, FSIM, visual results in terms of FSIM map.
\end{abstract}

Keywords: Image quality assessment, image comparison, color image quality, FSIM, S-CIELAB

\section{Introduction}

The image quality (IQ) assessment is one of the most important topics in image processing [1-4]. The IQ weights the received image distortion compare to the original image $[5,6]$. The imaging system can present some amounts of deformity in the original signal. Thus IQ measurement is significant. By clarifying the IQ, the IQ metrics become specialized with the terms of objective. However, the subjective feeling by human observation at an image is also important [7].

There are several goals that we use IQ metrics. One is how to measure the sharpness of given image $[8,9]$. This sharpness effect affects the quantity of detail an image. Another goal is to assess the amount of noise. The standard noise removal system lessens the amount of noise by averaging or smoothing an image. But this technique can sacrifice image details. Another goal is to assess dynamic range. The dynamic range is the range of intensity levels in a captured image. The dynamic range is highly related to the noise because high noise can cause low dynamic range. The tone reproduction can be measured with the relationship between scene brightness and the reproduced intensity. The color preciseness is measured in various objective tools. Finally, color moiré, color artifacts also can be assessed by IQ.

The goal of this study is to assess IQ without the association of human viewers [10-17]. The remainder of the manuscript is arranged as follows. In Section 2, we introduce various IQ metrics. In Section 3, we provide the assess IQ results in various metrics. Objective and subjective performances are assessed and compared. Finally, conclusion remarks are shown in Section 4.

\section{Image Quality Metrics}

The assessment of IQ measure regarding the apperception is an important point for guaranteeing that the metrics can replace and help human viewers in the IQ assessment. Thus, in this section, we introduce and analyze some existing IQ metrics. The conventional IQ metrics can be classified into several groups as shown in Table 1. 
Table 1. Image Quality Metrics and their Characteristics

\begin{tabular}{|c|c|}
\hline IQ metrics & Characteristics \\
\hline $\begin{array}{l}\text { Color Mean Squared Error } \\
\text { (CMSE) }\end{array}$ & $\begin{array}{l}\text { Mathematically calculated metric. This metric is computed by } \\
\text { the intensity of the distortions. }\end{array}$ \\
\hline $\begin{array}{l}\text { Color Peak Signal to Noise Ratio } \\
\text { (CPSNR) }\end{array}$ & $\begin{array}{l}\text { Mathematically calculated metric. This metric is computed by } \\
\text { the intensity of the distortions and MSE }\end{array}$ \\
\hline $\begin{array}{l}\text { Spatial-CIELAB } \\
{[18]}\end{array}$ & $\begin{array}{l}\text { Low-level-based IQ metric. This metric considers the } \\
\text { visibility of the deformity }\end{array}$ \\
\hline A Feature SIMilarity (FSIM) [19] & $\begin{array}{l}\text { High-level-based IQ metric. This metric quantifies image } \\
\text { quality based on the assumption that the human visual system } \\
\text { is conformed to squeeze information from the image. }\end{array}$ \\
\hline
\end{tabular}

The MSE is a mathematically calculated metric. The MSE is always non-negative, and values closer to zero are better. This computes the cumulative squared differences between the original signal and the restored signal. The MSE is computed as

$$
M S E=\frac{1}{m n} \sum_{j=0}^{m-1} \sum_{i=0}^{n-1}[E(i, j)]^{2}
$$

Here, parameters $i$ and $j$ stand for pixel location, $m$ and $n$ are image width and image height.

The peak signal-to-noise ratio (PSNR) is an engineering term for the ratio between the most possible power of an image and the power of restored image that influences the fidelity of its representation. The PSNR is normally represented in terms of the logarithmic decibel scale because PSNR is generally very high. The PSNR is calculated as

$$
P S N R=10 \log _{10}\left[\frac{p^{2}}{M S E}\right]
$$

Here, $p$ is the maximum possible pixel value of the image.

One of the most well-known color difference is CIELAB which is released by CIE. In this color space, it is easy to compute the difference between two given colors, by using the Euclidean distance - square root of MSE. The difference between two images is calculated as,

$$
\Delta E_{a b}^{*}=\sqrt{\left(\Delta L^{*}\right)^{2}+\left(\Delta a^{*}\right)^{2}+\left(\Delta b^{*}\right)^{2}}
$$

Here, $\Delta L^{*}=L_{o}^{*}-L_{r}^{*}, \Delta a^{*}=a_{o}^{*}-a_{r}^{*}$, and $\Delta b^{*}=b_{o}^{*}-b_{r}^{*}$.

In [18], Zhang and Wandell presented a spatial extension of the formula. Figure 1 is the flowchart of the S-CIELAB metric. There are two main steps in S-CIELAB: (1) spatial 
filtering to computing the blurring of the HVS, (2) persistence with the basic CIELAB computation for the uniform area. The RGB image is transformed into CIEXYZ and then $\mathrm{O}_{1}, \mathrm{O}_{2}$, and $\mathrm{O}_{3}$ color space by Eq. (4).

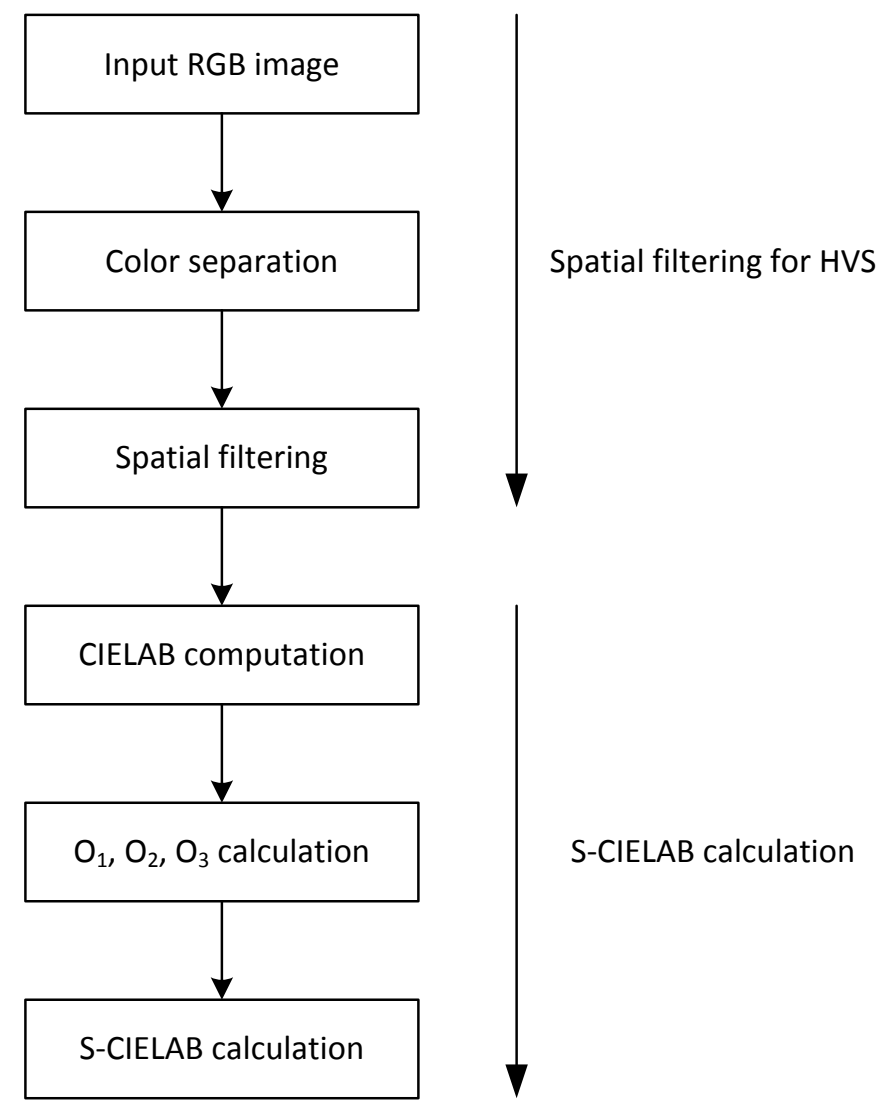

Figure 1. Flowchart of the S-CIELAB Calculation

$$
\begin{aligned}
& O_{1}=0.279 X+0.72 Y+0.107 Z, \\
& O_{2}=-0.449 X+0.29 Y-0.077 Z, \\
& O_{3}=0.086 X+0.59 Y+0.501 Z .
\end{aligned}
$$

The SSIM index tries to quantify the subjective difference between a deformed image and the reference one. SSIM explains the structural data in an image as those attributes which stand for the structure of the objects in the scene, independent of the mean luminance and contrast. The SSIM metric is based on a union of luminance, contrast, and structure comparison. The SSIM is calculated as

$$
\operatorname{SSIM}\left(\operatorname{im}_{1}, i_{2}\right)=\frac{\left(2 \mu_{x} \mu_{y}+c_{1}\right)\left(2 \sigma_{x} \sigma_{y}+c_{2}\right)}{\left(\mu_{x}^{2}+\mu_{y}^{2}+c_{1}\right)\left(\sigma_{x}^{2}+\sigma_{y}^{2}+c_{2}\right)}
$$

Here, $\mu$ is the average intensity for images $i m_{1}$ and $i m_{2}$, and $\sigma$ is the standard deviation of the images $i m_{1}$ and $i m_{2}$. Two parameters $c_{1}$ and $c_{2}$ are computed as $c_{1}=\left(K_{1} L\right)^{2}$ and $c_{2}=\left(K_{2} L\right)^{2}$. 


\section{Simulation Results}

In this Section, we present visual performance comparison for 20 images. They are obtained from LC dataset, \#101-120 images. The size of images are 720×540. Figure 2 shows tested 20 images. We used introduced four metrics for comparison. To compare the performance, we firstly reduced the size of test image with factor of $n(=2,4,8)$ and then restored it by enlarging the size.

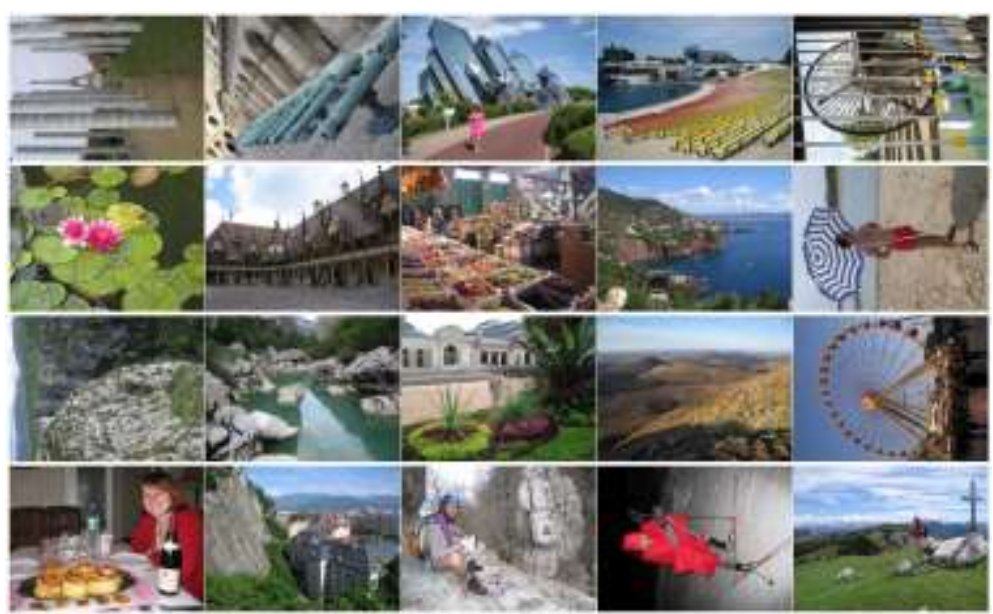

Figure 2. 20 Test Images from LC Dataset: \#101-120 Images

Figure 3-5 show three examples of image resizing. Firstly, all (a) images are original images with 512x512 size. Images (b) are obtained by factor of 2. Images (c) and (d) were obtained by factors of 4 and 8 . As one can see, as factor increase images became blurred.

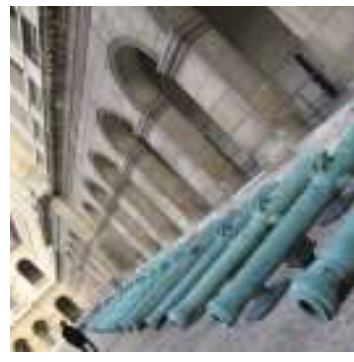

(a)

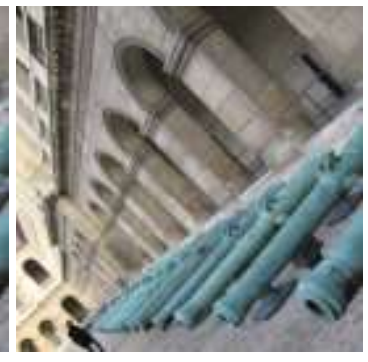

(b)

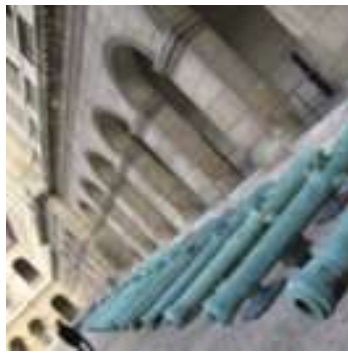

(c)

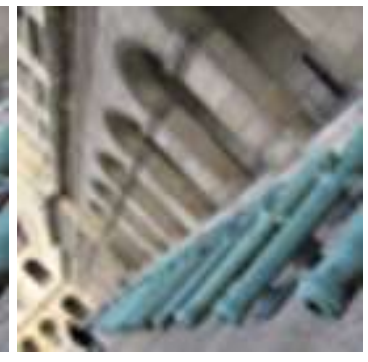

(d)

Figure 3. Test on \#102 Image: (a) Original Image, (b) Factor of 2, (c) Factor of 4 , and (d) Factor of 8

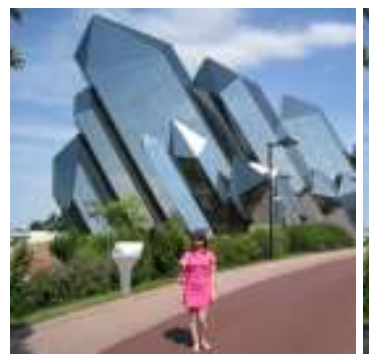

(a)

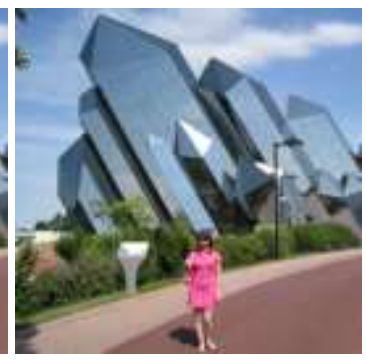

(b)

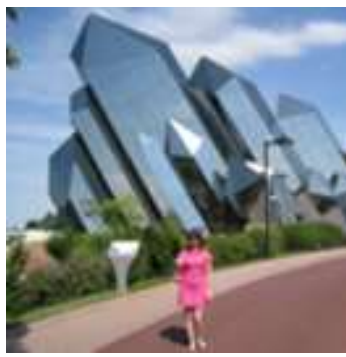

(c)

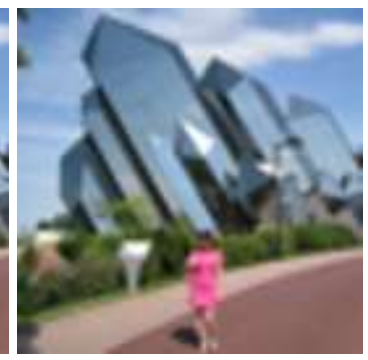

(d) 
Figure 4. Test on \#103 Image: (a) Original Image, (b) Factor of 2, (c) Factor of 4 , and (d) Factor of 8

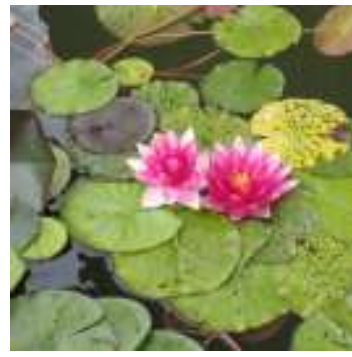

(a)

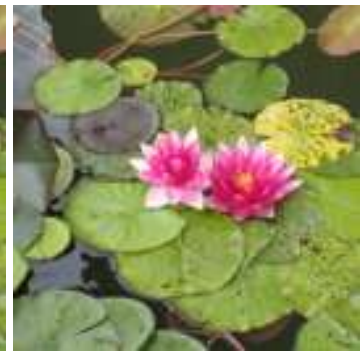

(b)

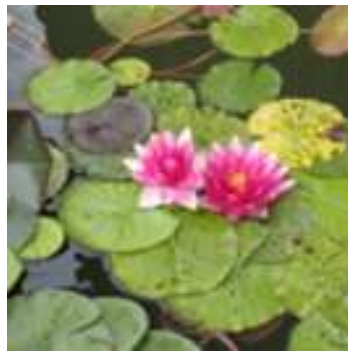

(c)

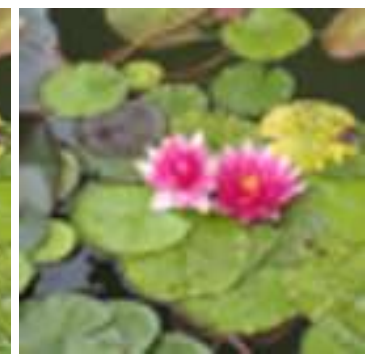

(d)

Figure 5. Test on \#106 Image: (a) Original Image, (b) Factor of 2, (c) Factor of 4, and (d) Factor of 8

Figures 6 and 7 show FSIM maps comparison. Figure 6(a) shows original image of \#120 LC image. Figures 6(b-d) display the result with factor of 2, 4, and 8.

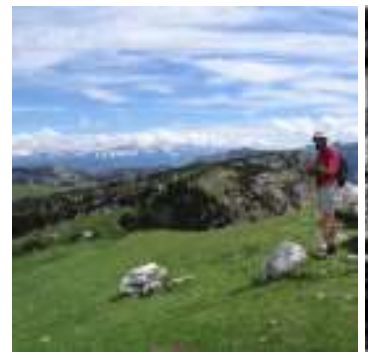

(a)

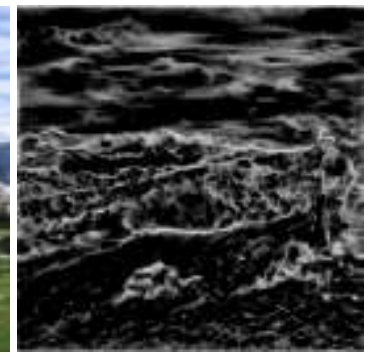

(b)

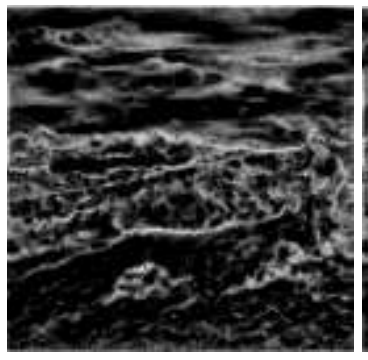

(c)

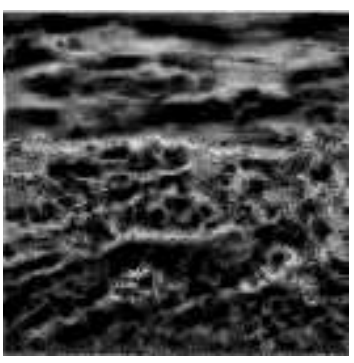

(d)

Figure 6. FSIM Comparison on \#120 Image: (a) Original Image, (b) Factor of 2, (c) Factor of 4, and (d) Factor of 8

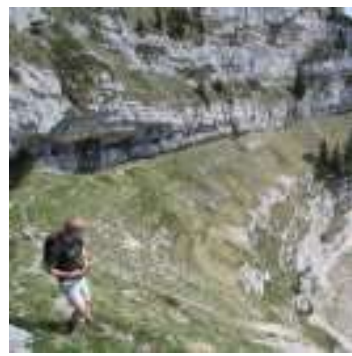

(a)

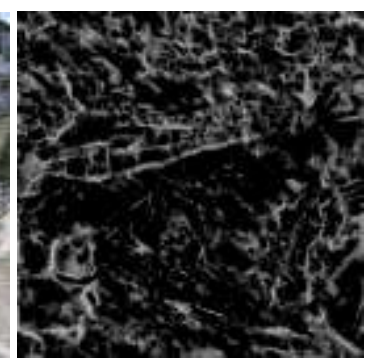

(b)

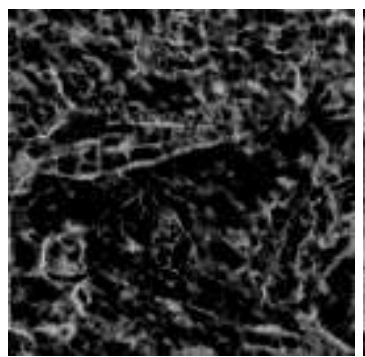

(c)

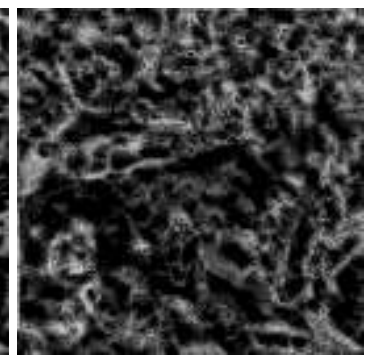

(d)

Figure 7. FSIM Comparison on \#121 Image: (a) Original Image, (b) Factor of 2, (c) Factor of 4, and (d) Factor of 8 
Table 2. Objective Performance Comparison using CMSE

\begin{tabular}{|c|c|c|c|}
\hline & factor $=2$ & factor $=4$ & factor $=8$ \\
\hline 101 & 43.157 & 102.649 & 207.357 \\
\hline 102 & 73.941 & 179.687 & 340.985 \\
\hline 103 & 77.858 & 173.655 & 322.380 \\
\hline 104 & 234.158 & 482.164 & 755.728 \\
\hline 105 & 244.618 & 692.206 & 1262.191 \\
\hline 106 & 63.473 & 175.936 & 349.668 \\
\hline 107 & 95.603 & 250.379 & 397.678 \\
\hline 108 & 269.859 & 605.132 & 1018.662 \\
\hline 109 & 107.448 & 232.490 & 380.355 \\
\hline 110 & 150.150 & 291.806 & 462.508 \\
\hline 111 & 159.702 & 371.454 & 654.308 \\
\hline 112 & 103.978 & 241.372 & 441.416 \\
\hline 113 & 222.471 & 518.483 & 819.393 \\
\hline 114 & 51.321 & 112.223 & 185.138 \\
\hline 115 & 180.205 & 414.471 & 659.163 \\
\hline 116 & 86.554 & 198.102 & 333.137 \\
\hline 117 & 119.671 & 248.988 & 396.517 \\
\hline 118 & 191.537 & 400.585 & 637.398 \\
\hline 119 & 31.981 & 101.833 & 218.126 \\
\hline 120 & 74.252 & 153.214 & 244.585 \\
\hline Avg. & 129.097 & 297.341 & 504.335 \\
\hline
\end{tabular}

For objective performance comparison, Tables 2-5 show results by four metrics. They are CMSE, CPSNR, S-CIELAB, and FSIM. Figure 8 depicts results of Tables 2-5.

Table 3. Objective Performance Comparison using CPSNR

\begin{tabular}{|c|c|c|c|}
\hline & factor $=2$ & factor $=4$ & factor $=8$ \\
\hline 101 & 31.780 & 28.017 & 24.964 \\
\hline 102 & 29.442 & 25.586 & 22.803 \\
\hline 103 & 29.218 & 25.734 & 23.047 \\
\hline 104 & 24.436 & 21.299 & 19.347 \\
\hline 105 & 24.246 & 19.728 & 17.120 \\
\hline 106 & 30.105 & 25.677 & 22.694 \\
\hline 107 & 28.326 & 24.145 & 22.135 \\
\hline 108 & 23.819 & 20.312 & 18.051 \\
\hline 109 & 27.819 & 24.467 & 22.329 \\
\hline 110 & 26.366 & 23.480 & 21.480 \\
\hline 111 & 26.098 & 22.432 & 19.973 \\
\hline 112 & 27.961 & 24.304 & 21.682 \\
\hline 113 & 24.658 & 20.983 & 18.996 \\
\hline 114 & 31.028 & 27.630 & 25.456 \\
\hline 115 & 25.573 & 21.956 & 19.941 \\
\hline 116 & 28.758 & 25.162 & 22.905 \\
\hline 117 & 27.351 & 24.169 & 22.148 \\
\hline 118 & 25.308 & 22.104 & 20.087 \\
\hline 119 & 33.082 & 28.052 & 24.744 \\
\hline 120 & 29.424 & 26.278 & 24.247 \\
\hline Avg. & 27.740 & 24.076 & 21.707 \\
\hline
\end{tabular}


Table 4. Objective Performance Comparison using S-CIELAB

\begin{tabular}{|c|c|c|c|}
\hline & factor $=2$ & factor $=4$ & factor $=8$ \\
\hline 101 & 1.12791 & 2.42533 & 4.42874 \\
\hline 102 & 1.54737 & 3.43755 & 5.97868 \\
\hline 103 & 1.62831 & 3.26345 & 5.53084 \\
\hline 104 & 3.19777 & 6.34246 & 9.95759 \\
\hline 105 & 3.27214 & 7.51711 & 12.73732 \\
\hline 106 & 2.18061 & 4.94536 & 8.31241 \\
\hline 107 & 1.82230 & 3.97636 & 5.83023 \\
\hline 108 & 3.87617 & 7.83984 & 12.44479 \\
\hline 109 & 2.02470 & 4.07238 & 6.31687 \\
\hline 110 & 1.95371 & 4.04639 & 6.68887 \\
\hline 111 & 2.58781 & 5.47537 & 8.73121 \\
\hline 112 & 1.86635 & 3.88038 & 6.48905 \\
\hline 113 & 3.54726 & 7.09699 & 10.37352 \\
\hline 114 & 1.41585 & 2.79602 & 4.30780 \\
\hline 115 & 2.51917 & 5.09198 & 7.63409 \\
\hline 116 & 1.92671 & 3.94229 & 6.37339 \\
\hline 117 & 2.49023 & 4.63612 & 6.88849 \\
\hline 118 & 2.66510 & 5.32236 & 8.16488 \\
\hline 119 & 0.99283 & 2.29512 & 3.87868 \\
\hline 120 & 1.85200 & 3.47395 & 5.28279 \\
\hline Avg. & 2.22472 & 4.59384 & 7.31751 \\
\hline
\end{tabular}

Table 5. Objective Performance Comparison using FSIM

\begin{tabular}{|c|c|c|c|}
\hline & factor $=2$ & factor $=4$ & factor $=8$ \\
\hline 101 & 0.99100 & 0.91486 & 0.80015 \\
\hline 102 & 0.99258 & 0.91250 & 0.79072 \\
\hline 103 & 0.99108 & 0.91203 & 0.79542 \\
\hline 104 & 0.98168 & 0.86161 & 0.71348 \\
\hline 105 & 0.98646 & 0.86776 & 0.70765 \\
\hline 106 & 0.99303 & 0.90777 & 0.77576 \\
\hline 107 & 0.98762 & 0.88855 & 0.76659 \\
\hline 108 & 0.98489 & 0.87233 & 0.70231 \\
\hline 109 & 0.98809 & 0.88707 & 0.73805 \\
\hline 110 & 0.98491 & 0.85288 & 0.71916 \\
\hline 111 & 0.98984 & 0.89361 & 0.70984 \\
\hline 112 & 0.99069 & 0.90726 & 0.77924 \\
\hline 113 & 0.98336 & 0.86113 & 0.70591 \\
\hline 114 & 0.99243 & 0.91647 & 0.78697 \\
\hline 115 & 0.98426 & 0.83751 & 0.68200 \\
\hline 116 & 0.98981 & 0.90523 & 0.78382 \\
\hline 117 & 0.98695 & 0.88651 & 0.73713 \\
\hline 118 & 0.98639 & 0.87943 & 0.72001 \\
\hline 119 & 0.99319 & 0.91545 & 0.79362 \\
\hline 120 & 0.99227 & 0.91830 & 0.79853 \\
\hline Avg. & 0.98853 & 0.88991 & 0.75032 \\
\hline
\end{tabular}


International Journal of Signal Processing, Image Processing and Pattern Recognition Vol. 10, No. 8 (2017)

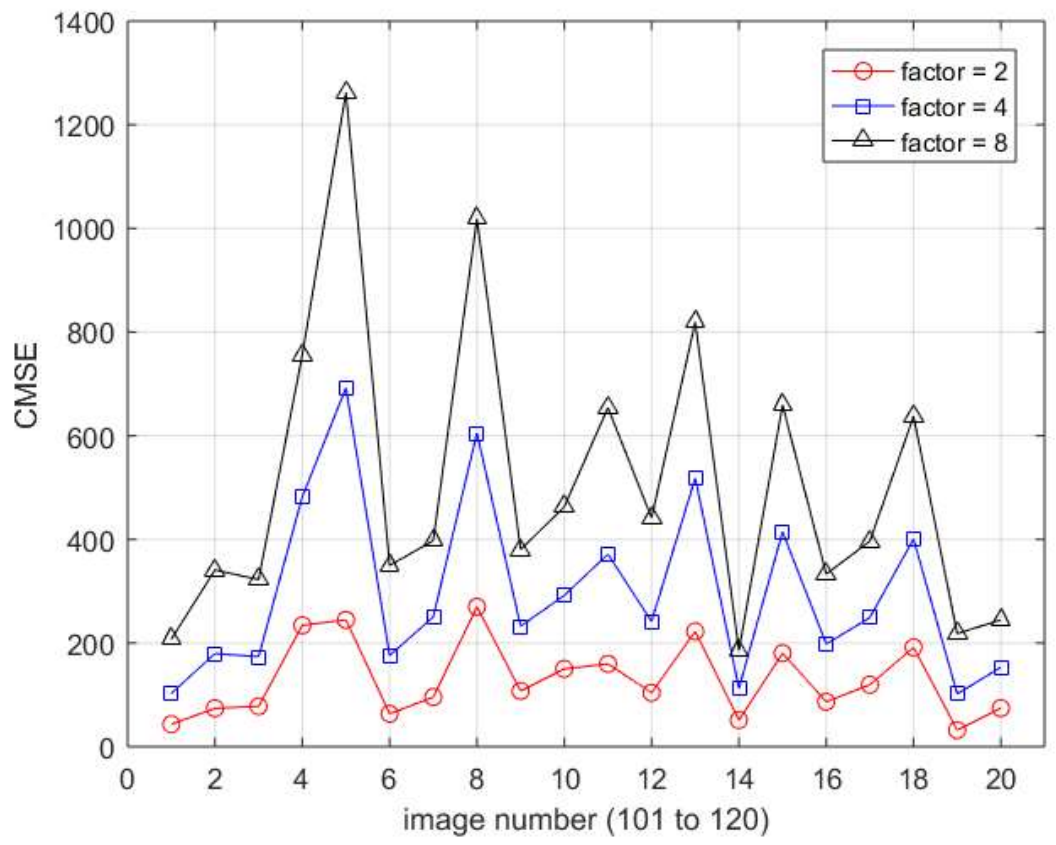

(a)

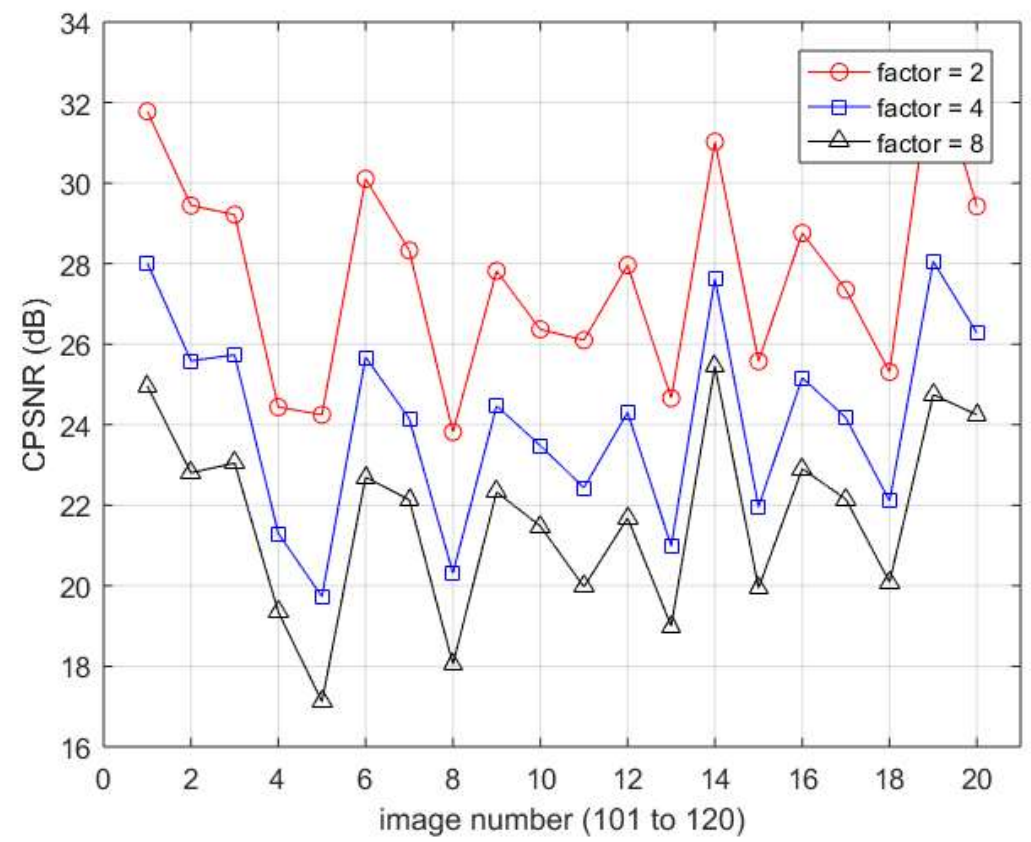

(b) 


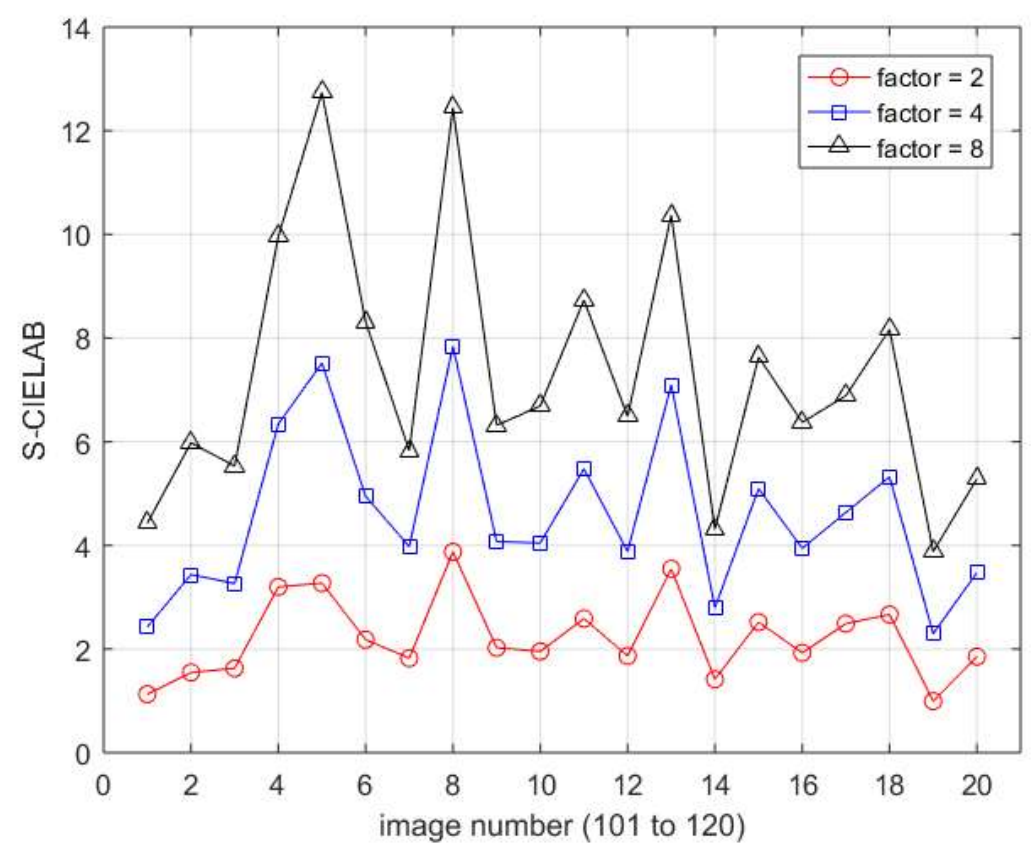

(c)

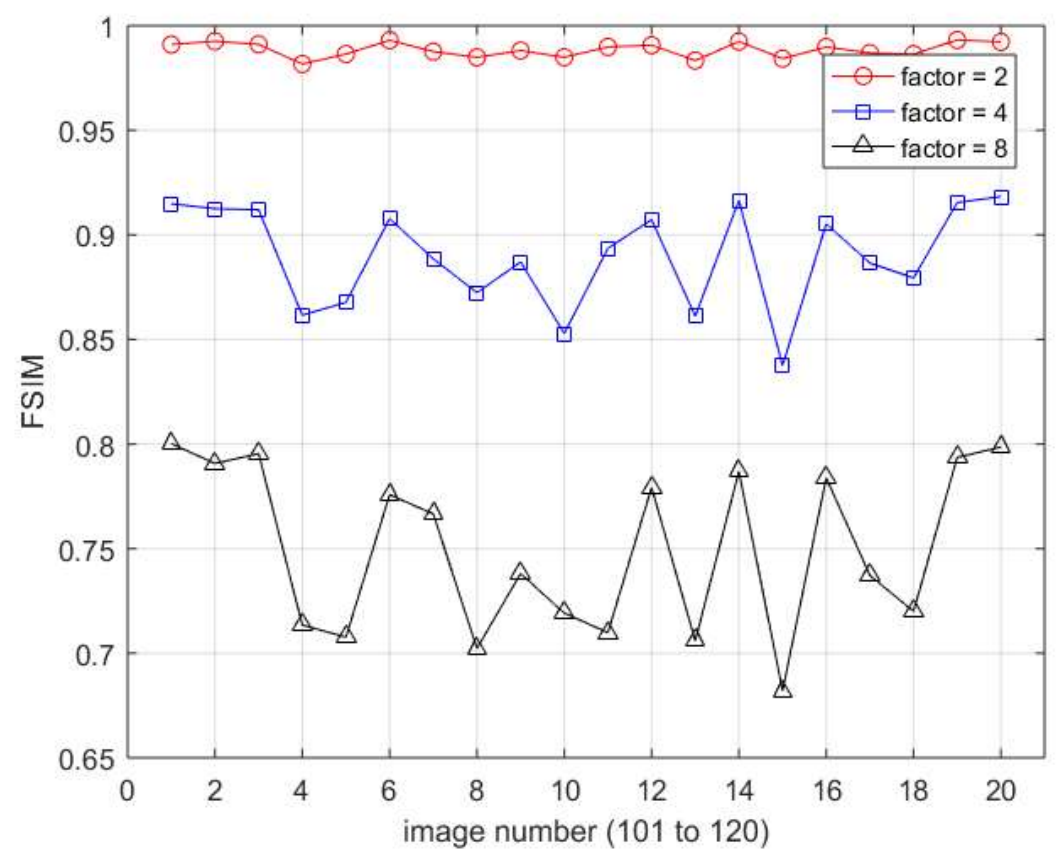

(d)

Figure 8. Depicted Performances: (a) CMSE, (b) CPSNR, (c) S-CIELAB, and (d) FSIM

\section{Conclusions}

Image quality assessment is an important topic. The image quality weights the received image distortion compare to the original one. The goal of this study is to assess image quality without the association of human viewers. Four objective performance metrics are described, and they are used for image comparison. Three factor values were used in the 
simulation. The results are shown in several ways, numerical results in terms of CMSE, CPSNR, S-CIELAB, FSIM, visual results in terms of FSIM map.

\section{Acknowledgements}

This work was supported by the National Research Foundation of Korea(NRF) Grant funded by the Korean Government (2015R1D1A1A01058171). This paper is a revised and expanded version of a paper entitled "Quality Assessment Using Metrics" presented at SIP2016.

\section{References}

[1] S. Hu, L. Jin, H. Wang, Y. Zhang, S. Kwong, and C. -C. Jay Kuo, "Compressed image quality metric based on perceptually weighted distortion," IEEE Transactions on Image Processing, vol. 24, no. 12, pp. 5594-5608, 2015.

[2] P. Prijitha and N. Naveen, "Gradient and luminance similarity based image quality metric," in Proc. ICCCI, 2015.

[3] C. Galkandage, J. Calic, V. De Silva, and S. Dogan, "A full-reference stereoscopic image quality metric based on binocular energy and regression analysis," in Proc. 3DTV-CON, pp. 1-5, 2015.

[4] A. S. Imran and F. A. Cheikh, "Blind image quality metric for blackboard lecture images," in Proc. Signal Processing Conference, pp. 333-337, 2010.

[5] C. Charrier and T. Eude, "A psychovisual color image quality metric integrating both intra and inter channel masking effect," in Proc. Signal Processing Conference, pp. 2123-2126, 2004.

[6] A. Chetouani, A. Beghdadi, M. Deriche, A. Bouzerdoum, "A Reduced Reference Image Quality Metric based on feature fusion and neural networks," in Proc. Signal Processing Conference, pp. 589-593,

[7] A. Chetouani, "Full reference image quality metric for stereo images based on Cyclopean image computation and neural fusion," in Proc. Visual Communications and Image Processing Conference, pp. 109-112, 2014.

[8] S. A. Fezza, M.-C. Larabi, K. M. Faraoun, Stereoscopic image quality metric based on local entropy and binocular just noticeable difference, in Proc. IEEE International Conference on Image Processing, 2014.

[9] K. Gu, G. Zhai, X. Yang, and W. Zhang, "An efficient color image quality metric with local-tunedglobal model," in Proc. IEEE International Conference on Image Processing, pp. 506-510, 2014.

[10] X. Chen, G. Jeon, and J. Jeong, “A filter switching interpolation method for deinterlacing,” SPIE Optical Engineering, vol. 51, no. 10, pp. 107402, October 2012.

[11] S.J. Park, G. Jeon, and J. Jeong, "Covariance-based adaptive deinterlacing method using edge map," in Proc. IEEE IPTA2010, Paris, France, July 7-10 2010, pp. 166-171.

[12] S.J. Park, G. Jeon, and J. Jeong, "Deinterlacing algorithm using edge direction from analysis of the DCT coefficient distribution," IEEE Trans. Consumer Electronics, vol. 55, no. 3, pp. 1674-1681, Aug. 2009.

[13] G. Jeon, S.J. Park, Y. Fang, M. Anisetti, V. Bellandi, E. Damiani, and J. Jeong, "Specification of efficient block matching scheme for motion estimation in video compression," SPIE Optical Engineering, vol. 48, no. 12, Dec. 2009, pp. 127005.

[14] J. Wu, Z. Xu, G. Jeon, X. Zhang, and L. Jiao, "Arithmetic coding for image compression with adaptive weight-context classification," Signal Processing: Image Communication, vol. 28, no. 7, pp. 727-735, Aug. 2013.

[15] X. Chen, G. Jeon, and J. Jeong, "Voting-based directional interpolation method and its application to still color image demosaicking," IEEE Trans. Circuits and Systems for Video Technology, vol. 24, no. 2, pp. 255-262, February 2014.

[16] X. Chen, G. Jeon, J. Jeong, and L. He, "Multidirectional weighted interpolation and refinement method for Bayer pattern CFA demosaicking," IEEE Trans. Circuits and Systems for Video Technology, vol. 25, no. 8, pp. 1271-1282, Aug. 2015.

[17] J. Wang, G. Jeon, and J. Jeong, "Efficient adaptive de-Interlacing algorithm with awareness of closeness and similarity," SPIE Optical Engineering, vol. 51, no. 1, pp. 017003, January 2012.

[18] X. Zhang and B. A. Wandell, "A spatial extension of CIELAB for digital color image reproduction," In Soc. Inform. Display 96 Digest, pp. 731-734, San Diego,CA, 1996.

[19] L. Zhang, L. Zhang, X. Mou and D. Zhang, "FSIM: A Feature Similarity Index for Image Quality Assessment,” IEEE Trans. Image Processing, vol. 20, no. 8, pp. 2378-2386, 2011. 


\begin{abstract}
Author
Gwanggil Jeon received the BS, MS, and $\mathrm{PhD}$ (summa cum laude) degrees in Department of Electronics and Computer Engineering from Hanyang University, Seoul, Korea, in 2003, 2005, and 2008, respectively. From 2008 to 2009, he was with the Department of Electronics and Computer Engineering, Hanyang University, from 2009 to 2011, he was with the School of Information Technology and Engineering (SITE), University of Ottawa, as a postdoctoral fellow, and from 2011 to 2012, he was with the Graduate School of Science \& Technology, Niigata University, as an assistant professor. $\mathrm{He}$ is currently an associate professor with the Department of Embedded Systems Engineering, Incheon National University, Incheon, Korea. His research interests fall under the umbrella of image processing, particularly image compression, motion estimation, demosaicking, and image enhancement as well as computational intelligence such as fuzzy and rough sets theories. He was the recipient of the IEEE Chester Sall Award in 2007 and the 2008 ETRI Journal Paper Award.
\end{abstract}


International Journal of Signal Processing, Image Processing and Pattern Recognition Vol. 10, No. 8 (2017) 